\title{
Enfermedades periodontales y periimplantarias. Factores de riesgo y su diagnóstico
}

\author{
ECHEVERRÍA GARCÍA JJ *
}

\begin{abstract}
Echeverría García JJ. Enfermedades periodontales y periimplantarias. Factores de riesgo y su diagnóstico. Av Periodon Implantol. 2003; 15, 3: 149-156.
\end{abstract}

\begin{abstract}
RESUMEN
Algunas de las enfermedades de los tejidos que rodean a los dientes y a los implantes oseointegrados son infecciones producidas por una combinación de especies bacterianas que desbordan la capacidad de resistencia de los tejidos. En este sentido, la presencia de las respectivas enfermedades precisa no solamente de la presencia de una flora bacteriana adecuada sino también de una serie de factores de riesgo relacionados con el huésped y ambientales que determinan una respuesta tisular específica. Dentro de estos factores de riesgo deben considerarse, y hay que procurar identificar, si es posible, factores como la predisposición genética, ciertas enfermedades como la diabetes, el tabaco y la higiene oral. En el caso de la periimplantitis, no existe suficiente evidencia como para determinar tan claramente el papel de los factores de riesgo en su etiología y evolución clínica. Sin embargo, podrían ser similares a los mencionados para la periodontitis.
\end{abstract}

\section{PALABRAS CLAVE}

Enfermedades periodontales. Periodontitis. Periimplantitis. Factores de riesgo.

\section{INTRODUCCIÓN}

Las enfermedades periodontales son un grupo de enfermedades de carácter infeccioso que afectan a los tejidos que rodean y sostienen a los dientes. Hasta la década de los años setenta del siglo pasado se consideró a las infecciones periodontales como una sola entidad clínica, que se iniciaba en forma de gingivitis, y, en ausencia de tratamiento, progresaba de forma continua a periodontitis y finalmente, pérdida de los dientes. La causa de la enfermedad periodontal se creía que era el acúmulo inespecífico de placa y cálculo, y por lo tanto, su prevención y control se basaba exclusivamente en la eliminación de la máxima cantidad posible de ambos. Se consideraba que la enfermedad periodontal afectaba en mayor o menor grado prácticamente al $100 \%$ de la población, que todo el mundo era susceptible a ella, y que el grado de afectación dependía de la edad y de la higiene oral del individuo (1). Sin embargo, sobre la base de nuestros conocimientos actuales, se puede indicar que:
1. Sólo una pequeña proporción de la población experimenta periodontitis grave que compromete la supervivencia de los dientes. Por el contrario, la gingivitis y periodontitis moderadas son muy comunes, y compatibles con una buena función de la dentición hasta edades avanzadas.

2. La placa bacteriana asociada a la gingivitis y la periodontitis presenta similaridades y diferencias. La gingivitis precede a la periodontitis, pero sólo una fracción de las localizaciones gingivales con gingivitis sufrirá pérdida de soporte.

3. La periodontitis no es una consecuencia de la edad, aunque al ser una enfermedad crónica, en ausencia de tratamiento su gravedad aumenta al aumentar la edad del individuo.

4. La periodontitis no es la mayor causa de pérdida de dientes en adultos, excepto en poblaciones con bajos índices de caries y sin acceso a servicios dentales donde se extraigan dientes por otras razones (1).

\footnotetext{
* Catedrático.de Periodoncia. Facultad de Odontología. Universidad de Barcelona.
} 


\section{DEFINICIONES}

Los nuevos conceptos sobre la susceptibilidad a la periodontitis, han llevado a intentar conocer cuales son los grupos e individuos con un riesgo elevado de padecer la enfermedad. Antes de describir los diferentes factores que condicionan el nivel de riesgo de padecer enfermedad periodontal destructiva, y los métodos utilizados para la identificación de los grupos e individuos de riesgo elevado, es necesario definir varios conceptos que se manejan en la determinación del riesgo de enfermedad $(2,3)$.

\section{Factores de riesgo}

Son las características ambientales o individuales que cuando están presentes resultan directamente en un aumento de probabilidades de que la persona padezca la enfermedad, y que cuando están ausentes resultan directamente en una disminución de dichas probabilidades. Los factores de riesgo son características que se consideran etiológicas a la luz del conocimiento que se tiene en cada momento sobre la enfermedad de interés. Los factores de riesgo pueden ser de dos tipos, modificables (por ejemplo, los niveles de bacterias patógenas) e inmutables (por ejemplo, las características genéticas) La exposición a un factor de riesgo significa que éste debe existir previamente al inicio de la enfermedad, aunque la exposición puede ser continua, única o repetirse varias veces en un periodo de tiempo. La eliminación del factor de riesgo no significa que la enfermedad se cure, pero si que se reduzca la posibilidad de aparición. Del mismo modo, si una enfermedad tiene múltiples factores de riesgo, la eliminación de uno de ellos reducirá de forma proporcional la posibilidad de tenerla.

Hay que distinguir los factores de riesgo de otras características inmutables, pero que no se consideran etiológicas, y que pueden actuar como factores de confusión o pueden modificar los factores de riesgo. En esta categoría están características como la edad, el sexo y la raza.

\section{Marcadores de riesgo}

Son características asociadas a la enfermedad, que pueden ser o no etiológicas, y que puede utilizarse como indicadores de la presencia de enfermedad, como por ejemplo el sangrado al sondaje.

\section{Predictores de riesgo}

Son marcadores biológicos indicativos de la presencia de la enfermedad, de su historia pasada, o de su pro- gresión, pero que, a diferencia de los factores de riesgo, no son factores etiológicos. Un ejemplo de predictor de riesgo periodontal sería la pérdida de soporte.

\section{FACTORES DE RIESGO DE LAS PERIODONTITIS}

Según el concepto actual de la etiología multifactorial de las enfermedades periodontales, éstas se producen por la interacción de un agente microbiano, único o múltiple, un huésped más o menos susceptible, y unos factores ambientales que influyen sobre ambos.

\subsection{AGENTES MICROBIANOS}

La base científica para atribuir un papel etiológico en la destrucción periodontal a algunas especies bacterianas viene determinada por su presencia en mayores números y con mayor frecuencia en las localizaciones que presentan actividad, y su menor número o frecuencia en las inactivas, su disminución o desaparición cuando el tratamiento periodontal tiene éxito, y su reaparición en las lesiones recurrentes; el aumento de anticuerpos en suero y saliva contra esas especies; las manifestaciones de su virulencia, por ejemplo por la producción de sustancias citotóxicas, reabsorción ósea, etc.; y los estudios en animales. Todo ello reúne un cuerpo de evidencia muy considerable, que, en el caso de algunas especies, está siendo confirmado en estudios prospectivos.

Las principales bacterias que se considera tienen un papel causal en las periodontitis destructivas son: $A$. actinomycetemcomitans, $P$. gingivalis, $P$. intermedia, $E$. corrodens, $F$. nucleatum, $B$. forsythus, $C$. rectus y varias especies de espiroquetas o Treponema. Hay muchas más que se han implicado en la etiología de la destrucción periodontal, y el desarrollo de la investigación puede hacer que otras especies que no se han mencionado pasen a ocupar un papel principal, pero en este momento las especies citadas son las que más a menudo se asocian a las periodontitis (4).

Entre las especies que se han nombrado, hay estudios longitudinales que parecen demostrar sin duda el papel etiológico de $A$. actinomycentemcomitans en las periodontitis juveniles y prepuberales, aunque también se asocia a las periodontitis avanzadas del adulto y a las periodontitis refractarias. $P$. gingivalis se asocia principalmente a la pérdida de soporte en la periodontitis del adulto y la periodontitis refractaria, y su presencia se ha demostrado que es un factor de riesgo de actividad periodontal en estudios prospectivos, al igual que $P$. intermedia (5). 


\section{Diagnóstico bacteriano}

El conocimiento detallado de las especies bacterianas asociadas a diferentes condiciones del periodonto sólo tiene interés si sirve para predecir, prevenir o tratar los problemas periodontales y para monitorizar los resultados del tratamiento. Diversos estudios han encontrado valor como predictor de riesgo al aumento de las especies bacterinas señaladas anteriormente, junto a niveles bajos de especies protectoras como el Streptococcus sanguis II y Capnocytophaga ochracea. De todas estas bacterias, las más estudiadas como predictores de enfermedad son $A$. actinomycetemcomitans y $P$. gingivalis.

A pesar del gran interés de los marcadores y predictores bacterianos, es evidente que la obtención de estos indicadores no es sencilla, desde el punto de vista clínico o epidemiológico, e incluso en el laboratorio los periodontopatógenos anaerobios precisan técnicas de cultivo especiales y que toman un tiempo considerable. En los últimos años, se han desarrollado métodos muy sofisticados de identificación de especies subgingivales, como la inmunofluorescencia y las sondas genéticas, que permiten analizar directamente las muestras de placa, sin necesidad de prolongados cultivos, facilitando mucho la investigación en este campo (6). A fin de hacer factible el diagnóstico microbiológico periodontal en la clínica, se han desarrollado un número de pruebas de identificación microbiana rápida principalmente de tres tipos: pruebas enzimáticas, sondas de DNA y pruebas inmunológicas. Parece que es prematuro recomendar el uso de estas pruebas diagnósticas en la práctica diaria, aunque encierran una gran promesa para la predicción del pronóstico de la periodontitis destructiva, y por lo tanto es deseable su evaluación en muestras grandes de individuos con y sin enfermedad periodontal, en estudios longitudinales, y comparando los resultados con cultivos, u otras técnicas de laboratorio, a fin de averiguar que especies son los verdaderos indicadores de actividad periodontal, los predictores de futura pérdida de soporte, y los responsables de una respuesta estable al tratamiento $(7,8)$.

\section{Marcadores en saliva y en el fluido crevicular}

Los niveles de enzimas, proteínas, microorganismos e inmunoglobulinas secretoras en saliva se han estudiado en relación con las enfermedades periodontales. Algunos enzimas sólo se encuentran en saliva cuando el individuo está en una fase de actividad, y los anticuerpos IgA e IgG están elevados en algunos casos de periodontitis juvenil y del adulto, y disminuyen con el tratamiento. La accesibilidad y sencillez de recolección de la saliva la hace prometedora para pruebas de despistaje de enfermedad periodontal destructiva en poblaciones, pero todavía no se ha desarrollado ninguna que haya mostrado su utilidad en estudios longitudinales (9). El fluido crevicular se ha estudiado extensamente, y en él se detectan gran cantidad de indicadores de enfermedad periodontal destructiva (10). Un número considerable de estos productos parece tener capacidad para diferenciar entre gingivitis y enfermedad periodontal destructiva, para indicar una respuesta satisfactoria al tratamiento periodontal, e incluso para predecir futura pérdida de inserción (11).

\subsection{FACTORES DE SUSCEPTIBILIDAD DEL HUÉSPED}

\section{Factores genéticos y su diagnóstico}

La hipótesis sobre la presencia de factores de riesgo genéticos en el desarrollo de la periodontitis destructiva se originó en principio de la observación de una serie de síndromes, que se suponen determinados genéticamente, y que cursan con destrucción rápida y precoz de los tejidos periodontales (Papillon-Lefèvre, Down, Ehler-Danlos, etc.). En algunos casos las periodontitis asociada a estos síndromes se deben a un defecto que predispone a la destrucción rápida de los tejidos periodontales, como el del colágeno en el síndrome de Ehlers-Danlos, pero en la mayor parte de los síndromes congénitos que cursan con alteraciones periodontales se ha descrito defectos de la inmunidad, ya sea celular o humoral, siendo las disfunciones de los leucocitos polimorfonucleares (PMN) el hallazgo más frecuente.

Las periodontitis precoces (prepuberales y juveniles) que no se asocian a síndromes hereditarios son raras, pero se han descrito casos en los cuales se presentan aisladamente, y en los que el carácter congénito de este tipo de periodontitis se ha confirmado. En el caso de las periodontitis juveniles, está bien documentada su agrupación en familias, lo cual puede estar a favor de su carácter congénito, o de la presencia de factores ambientales comunes, sin embargo, los estudios realizados sobre este tipo de periodontitis descartan que su presencia en miembros de la misma familia se pueda deber solamente a una transmisión de $A$. actinomycetemcomitans. También se ha estudiado la asociación de periodontitis con el fenotipo HLA (human leukocyte antigen), y hay varios estudios que han encontrado una relación del fenotipo HLA A9 con la periodontitis juvenil, y algunos también con la periodontitis de evolución rápida y con la periodontitis del adulto. En general se considera que hay suficiente base científica a favor de la presencia de factores genéticos en las periodontitis de aparición temprana. Por lo que se refiere a la periodontitis del adulto, los estudios realizados en gemelos parecen indicar que 
los factores genéticos influencian la evolución de la enfermedad, aunque la evidencia es menos clara (12). En lo que respecta a la identificación de la posible predisposición genética, nos referiremos específicamente a las periodontitis agresivas en pacientes por otra parte sin enfermedades sistémicas que incluyan la presencia necesaria de periodontitis, como la mayoría de las mencionadas anteriormente. El papel que los genes pueden desempeñar en la periodontitis ha sido objeto de numerosas investigaciones en los últimos años. Se ha establecido que mucha de la variabilidad clínica que se observa en pacientes periodontales tiene una explicación genética (12) que se basa en la influencia de diferentes interleukinas sobre la reabsorción ósea y la destrucción del tejido conectivo. La producción de estas interleukinas (como la interleukina IL-1) estaría determinada y modulada por genes específicos. Se ha sugerido que aquellos pacientes positivos para IL-1 presentarían elevadas posibilidades de desarrollar periodontitis agresivas (13). Sin embargo, la correlación entre la presencia de IL-1 y el perfil de la enfermedad periodontal de un determinado paciente dista mucho de haber sido aclarada, y en consecuencia, el uso clínico de tests que detecten esta característica genética no está por ahora justificado en la identificación del paciente de riesgo de padecer periodontitis grave, ni debe servir para determinar el tratamiento ni las características del mantenimiento periodontales (14).

\section{Enfermedades sistémicas}

Las enfermedades sistémicas que se asocian a la periodontitis son de varios tipos, pero básicamente se pueden dividir en alteraciones secundarias de la inmunidad, como por ejemplo las leucemias y el SIDA; alteraciones endocrinas y metabólicas, como la diabetes y el embarazo y enfermedades inflamatorias como la colitis ulcerativa o enfermedad de Crohn.

Hay varias afecciones hematológicas que predisponen a la gingivitis severa y la periodontitis destructiva, y que se deben sospechar en un paciente que experimenta un súbito y considerable empeoramiento de su condición periodontal. Entre ellas están la anemia aplástica, la neutropenia de cualquier etiología, la leucemia aguda (principalmente mielomonocítica), los linfomas y las histiocitosis de células de Langerhans.

Entre las alteraciones metabólicas, la diabetes se ha relacionado tradicionalmente con las periodontitis, pero no todos los estudios de prevalencia de gingivitis y periodontitis en diabéticos coinciden en la mayor frecuencia de afectación gingival en estos pacientes. Su asociación es poco clara y parece depender de la edad, el tipo de diabetes, y el nivel de control de ésta a través de la dieta o de agentes hipoglucemiantes. En general hay acuerdo en que la diabetes predispone a una mayor gravedad de las enfermedades periodontales y viceversa, posiblemente debido al efecto sinérgico de un defecto inmunológico con alteración de la función de los PMN y un defecto metabólico.

Los cambios hormonales relacionados con la pubertad y el embarazo no son enfermedades, pero se incluyen en este apartado por el carácter sistémico de su relación con las enfermedades periodontales. La pubertad causa una predisposición temporal a la gingivitis cuyo mecanismo es poco claro, aunque en pacientes diabéticos se ha observado un cambio en la composición de la placa bacteriana al llegar a la pubertad que se asocia a gingivitis. En mujeres previamente libres de periodontitis se ha visto que el embarazo produce gingivitis severa e inicio de periodontitis destructiva, que se ha atribuido al efecto de los esteroides que aumentan durante el embarazo, o a una depresión de la inmunidad asociada a este estado de la mujer. La respuesta de la encía a los esteroides es una reacción exagerada a los irritantes locales, en especial a la placa bacteriana. Además la progesterona afecta el metabolismo del colágeno. En general los efectos del embarazo sobre la encía se controlan mediante una higiene oral especialmente cuidadosa. Más recientemente se han realizado estudios que parecen sugerir una relación entre la osteoporosis en mujeres postemenopausicas y la pérdida de dientes, aunque no hay evidencia concluyente de una relación directa entre dicha perdida y mayor severidad de la periodontitis.

\subsection{FACTORES AMBIENTALES}

Entre ellos destacan sobremanera la higiene oral y el tabaco $(15,16)$. Parece incuestionable que la gingivitis es una infección inespecífica causada por las bacterias de la placa supragingival, y asociada con el nivel de higiene oral. Asimismo, por el momento no se cuestiona el hecho de que la placa subgingival es una condición causal para el desarrollo de la periodontitis. No obstante, las encuestas epidemiológicas, especialmente las realizadas en los países en vías de desarrollo, han puesto claramente de manifiesto que los depósitos de placa y cálculo se correlacionan muy débilmente con la periodontitis severa, de forma que ésta es igualmente prevalente y grave en los países donde la higiene oral es una obligación social estricta, que en los países donde la higiene oral prácticamente no existe. Lo que esto significa es que la placa y el cálculo no pueden producir periodontitis en el individuo resistente, y que incluso mínimos niveles de ambos son capaces de producir destrucción periodontal grave en el individuo altamente susceptible. Esto no debe interpretarse en absoluto como una falta de relación entre los niveles de placa y cálculo y la 
periodontitis, ya que en aquellos que son moderadamente o altamente susceptibles, la destrucción periodontal será mayor cuanto peor sea la higiene oral. Los pacientes periodontales, son, por definición, susceptibles a la periodontitis, y por tanto las normas habituales de higiene oral personal, y mantenimiento profesional, están de acuerdo con la evidencia resultante de los datos epidemiológicos. En las personas susceptibles a la periodontitis destructiva, el acumulo de placa y cálculo es un factor de riesgo para la pérdida de soporte periodontal.

Otros factores como la dieta y nutrición, ciertos fármacos y especialmente el tabaco han mostrado su relación con las enfermedades periodontales $(9,17)$ En el caso del tabaco, numerosos estudios, tanto epidemiológicos como estudios clínicos controlados, han demostrado la relación entre el tabaco y la destrucción periodontal. La causa parece ser el efecto vasoconstrictor de la nicotina que, aunque da lugar a una disminución de la gingivitis y del fluido crevicular en los fumadores, produce a la vez una disminución de las defensas del huésped en el ámbito local. Durante mucho tiempo se atribuyó el aumento de periodontitis destructiva en los fumadores a factores relacionados con la higiene oral, pero actualmente está demostrado que, controlando por las variables de índice de placa y cálculo, los fumadores presentan mayor profundidad de sondaje, mayor destrucción ósea, y un incremento de la predisposición a la gingivitis necrotizante aguda. Hoy en día no hay ninguna duda de que el tabaco es un factor de riesgo de periodontitis destructiva, y el riesgo atribuible cuando se comparan fumadores con no fumadores es del orden de 2,5 a 6 veces mayor.

En lo que al estrés se refiere, su asociación con la gingivitis ulcero-necrótica se conoce desde hace tiempo, pero más recientemente se ha demostrado una asociación entre sucesos provocadores de sobrecarga emocional y las enfermedades periodontales, tanto periodontitis como gingivitis (18). Esta asociación es frecuente en otras enfermedades, y se supone ligada a mecanismos de inmunosupresión, con relación a su vez a la producción de hormonas como el cortisol, endorfinas, adrenalina, y catecolaminas (19).

\section{PERIIMPLANTITIS: FACTORES DE RIESGOY SU DIAGNÓSTICO}

Aunque numerosos estudios longitudinales demuestran la elevada tasa de supervivencia de implantes oseointegrados, se admite que un porcentaje variable de ellos se pierde a lo largo de los años.
La pérdida de un implante puede sobrevenir de manera precoz, es decir en el periodo entre su instalación y su carga, o bien tardíamente, es decir, una vez el implante ha sido cargado tras una satisfactoria oseointegración.

La pérdida tardía de un implante suele deberse a infección (periimplantitis) o a fallo mecánico por sobrecarga.

Se denomina periimplantitis a la inflamación que afecta a los tejidos que rodean a un implante oseointegrado funcional, produciéndose pérdida del hueso de soporte (20). Por lo tanto, esta definición excluye al fracaso del implante durante el periodo de integración, incluso por infección.

De acuerdo con Mombelli (21), la evidencia que señala el papel de las bacterias en la inflamación periimplantaria se ha demostrado a través de 1) estudios clínicos 2) diferencias en la placa bacteriana alrededor de tejidos periimplantarios sanos y enfermos 3) aplicación de ligaduras alrededor de implantes en animales, que retienen placa y producen cambios en la flora periimplantaria y, después, periimplantitis 4) tratamiento antimicrobiano que mejora clínicamente la periimplantitis 5) el hecho de que una adecuada higiene oral mejora el pronóstico a largo plazo de los implantes oseointegrados.

En la periimplantitis existen factores de riesgo identificables, pero a diferencia de lo que sucede en las periodontitis, no han sido todavía significativamente estudiados. Sin embargo, la flora bacteriana, el estado periodontal del paciente, el diseño del implante, así como factores ambientales (higiene oral, tabaco) y la presencia de enfermedades sistémicas podrían jugar un papel significativo en la génesis de la periimplantitis.

\subsection{FLORA BACTERIANA}

La flora bacteriana de la encía o mucosa alrededor de implantes donde no existe inflamación es muy similar a la flora bacteriana alrededor de dientes sin inflamación periodontal, es decir con mayoría de formas cocoides y gérmenes aerobios Gram+, mientras que la proporción de anaerobios Gram- es muy reducida. Por el contrario, la flora bacteriana alrededor de implantes con periimplantitis es muy parecida a la que se observa alrededor de dientes con periodontitis, incluyendo $A$. actinomycetemcomitans, $P$. gingivalis, $P$. intermedia, y otras especies periodontopatógenas. Tal composición se ha observado tanto en series de pacientes como en periimplantitis experimental en el modelo animal $(22,23)$. La procedencia de especies bacterianas alrededor de implantes con periimplanti- 
tis debe buscarse con toda probabilidad en la flora propia de dientes con periodontitis, que, a través de un mecanismo de traslación colonizan los tejidos blandos periimplantarios (24). En tal caso, habría que esperar que en pacientes totalmente desdentados portadores de implantes oseointegrados, la flora periodontal debería estar ausente, como así ha sido demostrado (25). Sin embargo, también se ha demostrado que existen especies bacterianas no periodontopatógenas alrededor de implantes, que proceden de otras localizaciones intraorales, como por ejemplo la lengua (26).

El diagnóstico de la presencia de microbios en el ámbito de la interfase tejido blando- implante será, por otra parte, muy similar a la que se ha mencionado en el caso de las periodontitis, incluyendo la ausencia de sangrado al sondaje como un indicador de salud periimplantaria y el uso de cultivos bacterianos y sondas DNA para determinar la presencia de determinadas especies bacterianas relacionadas con la periimplantitis, lo que, unido al correspondiente antibiograma puede ayudar a diseñar un adecuado protocolo terapéutico (21).

\subsection{ESTADO PERIODONTALY CARACTERÍSTICAS DE LA SUPERFICIE DEL IMPLANTE.}

El hecho de que en la mayoría de estudios la presencia de periimplantitis esté ligada a la existencia de una flora bacteriana típica de periodontitis, hace aconsejable eliminar la infección periodontal antes de proceder a la instalación de implantes, incluyendo si es necesario, la exodoncia de dientes con periodontitis avanzada (26). Sin embargo diferentes estudios sugieren que, incluso en pacientes que pierden soporte periodontal por periodontitis, el hueso periimplantario se mantiene relativamente estable (27), especialmente en el caso de implantes de superficie no rugosa en contacto con el tejido blando periimplantario $(28,29)$. Es necesario ser cuidadoso al analizar los resultados de los estudios sobre el efecto de la rugosidad de la superficie de un determinado implante en la estabilidad del hueso periimplantario: los implantes que presentan una superficie rugosa la presentan en el ámbito de la superficie en contacto con el hueso, mientras que la superficie del implante que se entiende en contacto con el tejido blando es lisa. Por lo tanto, si la superficie rugosa está contaminada por bacterias, éstas proceden en realidad de una superficie lisa (21). Lo que podría suceder es que, una vez alcanzada la superficie del implante en contacto con el hueso, puede ser que éste se destruya más rápidamente en implantes de superficie rugosa. Aunque en realidad todos los sistemas de implantes sean susceptibles a la periimplantitis (29), esta enfer- medad es muy poco prevalente, de manera que la mayoría de los fracasos tardíos de implantes oseointegrados tienen que ver con la calidad del hueso, su volúmen y sobrecargas oclusales (30).

\subsection{FACTORES AMBIENTALES Y ENFERMEDADES SISTÉMICAS.}

Como sucede en el paciente periodontal, la higiene oral y el tabaco se asocian a la pérdida de soporte óseo de los implantes oseointegrados $(31,32)$ mientras que el impacto de determinadas enfermedades sistémicas en la etiología de la periimplantitis está todavía poco documentado.

\section{CONCLUSIÓN}

En resumen, la consideración de los factores de riesgo, tanto en el caso de las periodontitis como en el de las periimplantitis, indica el papel fundamental de las bacterias como los factores etiológicos sine qua non de las respectivas infecciones. Sin embargo, las características clínicas de la respuesta del huésped a esta agresión bacteriana vienen determinadas de manera fundamental por la susceptibilidad de este mismo huésped - probablemente en relación con determinada carga genética - y por la influencia de factores ambientales, de los cuales el tabaco y la placa bacteriana parecen ser especialmente significativos.

\section{SUMMARY}

Infectious diseases of the tissues surrounding teeth and osseointegrated implants are produced by different bacterial species which overcome tissue defense mechanisms. In this regard, different individual as well as environmental risk factors would determine a specific tissue response to plaque accumulation. Some of these periodontal risk factors have been identified so far, e.g. genetic predisposition, diabetes, smoking and oral hygiene. Due to a lack of pertinent scientific evidence, it is difficult to enumerate which risk factors are involved in peri-implantitis, although could be very much similar to those related to periodontitis.

\section{KEY WORDS}

Periodontal diseases. Periodontitis. Peri-implantitis. Risk factors. 


\section{CORRESPONDENCIA}

José J. Echeverría García

Rda. G. Mitre, 174-176

08006 Barcelona

Tel.: 932124796

Fax: 934179266

e-mail: jjperio@teleline.es

\section{BIBLIOGRAFÍA}

1. Burt BA, Eklund SA. (1992) Dentistry, Dental Practice and the Community. $4^{a}$ ed. Filadelfia: WB Saunders, pp. 52-82, 113-28.

2. Beck JD. (1994) Methods of assessing risk for periodontitis and developing multifactorial models. Journal of Periodontology 65: 468-78.

3. Beck JD, Koch GG, Offenbacher S. (1995) Incidence of attachment loss over 3 years in older adults -new and progressing lesions. Community Dentistry and Oral Epidemiology 23: 291-6.

4. Genco RJ. ( 1996) Current view of risk factors for periodontal diseases. Journal of Periodontology 67: 1041-9.

5. Johnson NW. ( 1994) Risk factors and the diagnostic tests for destructive periodontitis. En: Lang NP y Karring T, eds. Proceedings of the lst European Workshop on Periodontology. London: Quintessence pp. 90-119.

6. Maiden MFJ, Carman RJ, Curtis MA, Gillett IR, Griffiths GS, Sterne JAC, Wilton JMA, Johnson NW. (1990) Detection of high-risk groups and individuals for periodontal diseases: laboratory markers based on the microbiological analysis of subgingival plaque. Journal of Clinical Periodontology 17:1-13.

7. Smith GLF. ( 1994) Diagnosis of periodontal disease activity by detection of key microbial antigens. Journal of Clinical Periodontology 21:615-20.

8. Wolff L., Dahlen G. y Aeppli D. (1994) Bacteria as risk markers for periodontitis. Journal of Periodontology. 64: 498510 .

9. Johnson NW. (1989) Detection of high-risk groups and individual for periodontal disease. International Dental Journal 39: 33-47.

10. Lamster I.B. (1992)The host response in gingival crevicular fluid: Potential applications in periodontitis clinical trials. Journal of Periodontology 63: 1117-23.

11. Offenbacher S, Soskolne WA, Collins JG. (1991)
Prostaglandins and other eicosanoids in gingival crevicular fluid as markers of periodontal disease susceptibility and activity. En: Johnson NW, ed. Risk markers for oral diseases. Volumen 3: Periodontal diseases. Cambridge: Cambridge University Press, pp. 313-37.

12. Michalowicz BS. (1994) Genetic and inheritance considerations in periodontal disease. Journal of Periodontology 65 (Suppl 5): 479-88.

13. Kornman K, Crane A, Wang HY, Di Giovini F.S. et al. (1997) The interleukin-1 genotype as a severity factor in adult periodontal disease. Journal of Clinical Periodontology 24: $72-7$

14. Greenstein G y Hart T (2002) Clinical utility of a genetic susceptibility test for severe chronic periodontitis. A critical evaluation. Journal of the American Dental Association 133: 452-9.

15. Löe H, Theilade E, Jensen SB. (1965) Experimental gingivitis in man. Journal of Periodontology 36: 177-87.

16. Baelum V, Manji F, Fejerskov O. (1991) The distribution of periodontal destruction in the populations of the nonindustrialized countries: Evidence for the existence of high risk groups and individuals. En: Johnson NW, ed. Risk markers for oral diseases. Volumen 3: Periodontal diseases. Cambridge: Cambridge University Press; pp. 27-75.

17. Scully C, Porte SR, Mutlu S. (1991) Changing, subjectbased risk factors for destructive periodontitis. En Johnson NW, ed. Risk markers for oral diseases. Volumen 3: Periodontal diseases. Cambridge: Cambridge University Press, pp. 139-78.

18. Croucher R, Marcenes WS, Torres MS, Hughes F, Sheiham A. (1997) The relationship between life-events and periodontitis. A case-control study. Journal of Clinical Periodontology 24: 39-43.

19. Loos B.G, Hamming H y van der Velden. (1998) Stress et Parodontites: Revue de Littérature. Journal de Parodontologie et d'implantologie Orale 17: 205-17.

20. Albrektsson T. e Isidor F. (1994) Consensus report of session IV. En: Proceedings of the 1st. European Workshop on Periodontology. Eds: Lang N.P. y Karring T. Quintesence, London pp. 365-9.

21. Mombelli A (1999) Prevention and therapy of periimplant infections. En Proceedings of the 3rd European Workshop on Periodontology. Implant Dentistry. Eds: Lang N, Karring T. Lindhe J. Quintessence Publishing Co. Inc. Berlin, pp. 281-303

22. Lang N, Brägger U, Walter D, Beamer B y Kornman K. 
(1993) Ligature-induced peri-implant infection cynomolgus monkeys. I. Clinical and radiographic findings. Clinical Oral Implants Research 4:2-11.

23. Leonhart A, Renvert S. y Dahlén G. (1999) Microbial findings at failing implants. Clinical Oral Implants Research 10: $339-345$.

24. Sanz M, Newman M.G., Nachnani S, Holt R, Stewart R y Flemmig T (1990). Characterization of the subgingival microbial flora around endosteal sapphire dental implants in partially edentulous patients. International Journal of Oral and Maxillofacial Implants 5: 247-53.

25. Danser M.M., van Winkelhoff A.J. y van der Velden U. (1997) Periodontal bacteria colonizing oral mucous membranes in edentolous patients wearing dental implants. Journal of Periodon-tology 68: 209-16.

26. Lee K.H., Tañer A.C., Maiden M.F. y Weber H.P. (1999) Pre- and post- implantation microbiota of the tongue, teeth, and newly-placed implants. Journal of Clinical Periodontology 26: 822-32.

27. van Steenberghe D., Naert I., Jacobs R. Y Quirynen M. (1997) Influence of inflammatory reactions vs. occlusal loading on peri-implant marginal bone level. Advances in Dental Research 13: 130-5.

28. Ellegaard B., Baelum V. Y Karring T. (1997) Implant therapy in periodontally compromised patients. Clinical Oral Implant Research 8: 180-8.
29. Quirynen M, Peeters W, Naert I, Coucke W y van Steenberghe D. (2001) Peri-implant health around screwshaped c.p. titanium machind implants in partially edentulous patients with or without ongoing periodontitis. Clinical Oral Implant Research 12: 589-94.

30. Tillmans H.W., Hermann J.S., Tiffee J.C., Burgess A.V. y Meffert R.M. (1998) Evaluation of three different dental implants in ligature induced peri implantitis in the beagle dog. Part II. Histology and microbiology. International Journal of Oral and Maxillofacial Implants 13: $59-68$.

31. Esposito M, Hirsch J.M., Lekholm U.Y Thomsen P. (1998) Biological factors contributing to failures of osseointegrated oral implants. (I). Success criteria and epidemiology. European Journal of Oral Sciences 106: 527-51.

32. Lindquist L.W., Rockler, B. y Carlsson G.E. (1988) Bone resorption around fixtures in edentolous patients treated with mandibular fixed tissue-integrated prostheses. Journal of Prosthetic Dentistry 59: 59-63.

33. Lindquist L.W., Carlsson G.E. y Jemt T. (1997) Association between marginal bone loss around osseointegrated mandibular implants and smoking habits: a 10-year follow-up study. Journal of Dental Research 76: 16671674. 